\title{
Immunotherapy in Some Types of Tumors
}

\author{
Dra Mirta D Ambra* \\ Chairman World Academy Medical Sciences, Especialist Ministry Health University Buenos Aires, Argentina
}

Received: 眥 May 28, 2018; Published: 眥 June 08, 2018

*Corresponding author: Dra Mirta D Ambra, Chairman World Academy Medical Sciences, Especialist Ministry Health University Buenos Aires, Argentina

\section{Charasteristics}

a) Immunotherapy exerts its antitumor action by stimulating the response immune response of patients to cancer, unlike classic treatments, which directly attack the tumor

b) Previously immunotherapy was limited to patients in whom the conventional treatment, usually with chemotherapy, but currently in patients with some types of tumors, such as melanoma or some lung cancers, is already considered the treatment of first choice

c) Immunotherapy is able to control some types of malignant tumors prognosis very long, even for several years

d) Immunotherapy exerts its antitumor action by stimulating the immune response of patients against cancer, unlike classic treatments, which attack directly to the tumor. This implies a series of advantages and characteristics of this novelty strategy.

e) Its main advantage is its ability to control the tumor for very long periods of time in a certain percentage of patients, which varies according to the type of cancer. In some patients with tumors that were previously considered incurable, at this time they are getting very long survivals, even years.

f) Currently, immunotherapy with antibodies that block PD-1 receptors or action on these PD-L1 protein receptors has shown efficacy against a large number of tumors, including among others melanoma, cancers of the lung, kidney, bladder, these treatments are usually administered intravenously and their toxicity is usually lower than conventional treatments, such as chemotherapy.

g) However, between a 5-15\% of patients can develop relevant toxicities, which are usually due to the activation of the immune system against the patient's own organism. The organs most frequently affected by these reactions are: the lung ("pneumonitis"), which manifests in the form of cough and shortness of breath; and the digestive tract ("colitis"), which presents as diarrhea. When they are used as unique drugs, which is the most usual Now a days, toxicity is not usually a major problem. However, when they use in combination, their frequency and severity is greater. New immunotherapy strategies Despite these results, there is still a long way to go, given that today Only $40-60 \%$ of melanoma patients benefit from these treatments between 10 and $30 \%$ of patients with other types of tumors.

Some of the main ones Developing strategies to improve the effectiveness of immunotherapy are:

a) Combination Immunotherapy: During the development of a tumor it is they can alter several phases of the immune response. Therefore, the use simultaneous treatment of two or more immunotherapy treatments is one of the strategies more used to increase antitumor efficacy. The combinations of immunotherapy have shown significant activity in patients with melanoma and renal cancer. The main mechanisms of action of drugs which are used for these combinations are: directly activate the answer immunological; unlock the inhibition of the immune response produced by many tumors; or provide fundamental elements to trigger the immune response, as antigens or cells of the immune system New vaccines: antitumor vaccines consist of administering patient tumor antigens (small fragments of it, usually proteins), for the immune system to recognize them and thus put in place the antitumor immune response. Modern molecular biology techniques have allowed to advance a lot in the processes of selection of antigens with greater possibilities of triggering these responses and, therefore, this is one of the most hopeful ways for the development of new treatments of immunotherapy. Currently there is an anti-cancer vaccine against cancer prostate whose use in patients is approved in the USA. (Sipuleucel). In addition, some vaccines against infectious diseases can confer a high degree of protection against tumors associated with them (for example: human papilloma virus, associated with cervical cancer, or hepatitis B virus, associated with hepatocarcinoma). 
Vaccination against these viral infections dramatically reduces the incidence of associated tumors.

b) CAR-T Cells (Chimeric Antigen Receptor, or Antigenic Receptor Chimeric): It consists of extracting the patient's immune cells; process them in the laboratory to express an antigen that specifically recognizes to tumor cells; and readminister them to the patient, to attack the tumor. This strategy is having considerable efficacy in patients with some types of leukemia, although its use in patients with solid tumors It seems more complicated. In addition, it is associated with relevant toxicities, although the Most can be controlled with specialized medical care. As we have already seen, the small advances, taken together, are relevant. From here our motto from SEOM: In Oncology, each advance is written in capital letters. These small advances, considered each of them in isolation, could have have been considered of little relevance, but accumulated among themselves have led to change in many cases in a remarkable way the prognosis and the quality of life of many patients. In oncology, each advance is written with capital letters (madrid, february 19, 2018).

c) CAR-T cells (Chimeric Antigen Receptor, or Antigen Receptorchimeric): it consists of extracting the patient's immune cells; process them in the laboratory to express an antigen that specifically recognizes to tumor cells; and readminister them to the patient, to attack the tumor. This strategy is having considerable efficacy in patients with some types of leukemia, although its use in patients with solid tumors. It seems more complicated.
CC (i) This work is licensed under Creative To Submit Your Article Click Here: Submit Article

DOI: $10.32474 /$ OAJOM.2018.01.000125

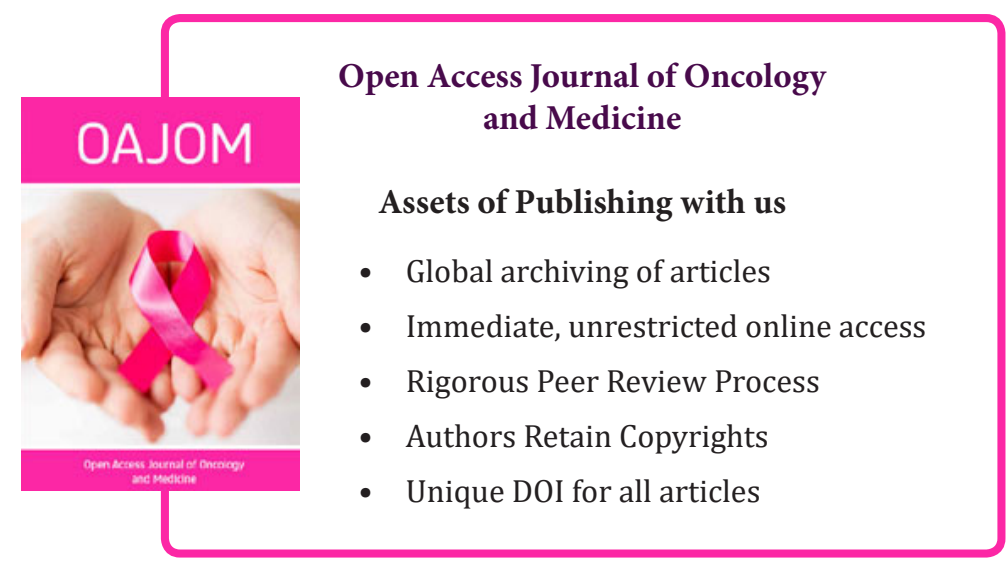

